University of New Hampshire

University of New Hampshire Scholars' Repository

Space Science Center

Institute for the Study of Earth, Oceans, and

Space (EOS)

2001

\title{
COMPTEL gamma-ray observations of the C4 solar flare on 20 January 2000
}

\author{
C A. Young \\ Goddard Space Flight Center \\ M B. Arndt \\ Bridgewater State College \\ K Bennett \\ ESTEC \\ A Connors \\ Eureka Scientific \\ H Debrunner \\ University of Berne
}

See next page for additional authors

Follow this and additional works at: https://scholars.unh.edu/ssc

Part of the Astrophysics and Astronomy Commons

\section{Recommended Citation}

COMPTEL gamma-ray observations of the C4 solar flare on 20 January 2000 Young, C. A. and Arndt, M. B. and Bennett, K. and Connors, A. and Debrunner, H. and Diehl, R. and McConnell, M. and Miller, R. S. and Rank, G. and Ryan, J. M. and Schoenfelder, V. and Winkler, C., AIP Conference Proceedings, 587, 613-617 (2001), DOI:http://dx.doi.org/10.1063/1.1419472

This Conference Proceeding is brought to you for free and open access by the Institute for the Study of Earth, Oceans, and Space (EOS) at University of New Hampshire Scholars' Repository. It has been accepted for inclusion in Space Science Center by an authorized administrator of University of New Hampshire Scholars' Repository. For more information, please contact Scholarly.Communication@unh.edu. 


\section{Authors}

C A. Young, M B. Arndt, K Bennett, A Connors, H Debrunner, R Diehl, Mark L. McConnell, R S. Miller, G Rank, James M. Ryan, V Schonfelder, and C Winkler 


\section{AIP $\mid$ proceedings}

\section{COMPTEL gamma-ray observations of the C4 solar flare on 20 January 2000}

C. A. Young, M. B. Arndt, K. Bennett, A. Connors, H. Debrunner, R. Diehl, M. McConnell, R. S. Miller, G. Rank, J. M. Ryan, V. Schoenfelder, and C. Winkler

Citation: AIP Conference Proceedings 587, 613 (2001); doi: 10.1063/1.1419472

View online: http://dx.doi.org/10.1063/1.1419472

View Table of Contents: http://scitation.aip.org/content/aip/proceeding/aipcp/587?ver=pdfcov

Published by the AIP Publishing

\section{Articles you may be interested in}

GLAST Measurements of PionDecay and Neutron Emission in Solar Flares

AIP Conf. Proc. 921, 468 (2007); 10.1063/1.2757405

Accelerated Particle Properties in Solar Flares from GammaRay Line Observations

AIP Conf. Proc. 891, 254 (2007); 10.1063/1.2713525

X- and gamma-ray observations of the 15 November 1991 Solar Flare

AIP Conf. Proc. 587, 618 (2001); 10.1063/1.1419473

Solar gamma-ray physics comes of age

AIP Conf. Proc. 587, 603 (2001); 10.1063/1.1419471

Gamma-ray astronomy: Promise for the future

AIP Conf. Proc. 387, 25 (1997); 10.1063/1.52001 


\title{
COMPTEL Gamma-Ray Observations of the C4 Solar Flare on 20 January 2000
}

\author{
C. A. Young ${ }^{\mathrm{a}}$, M. B. Arndt $\mathrm{t}^{\mathrm{b}}$, K. Bennett $\mathrm{c}^{\mathrm{c}}$, A. Connors ${ }^{\mathrm{d}}$, H. Debrunner ${ }^{\mathrm{e}}$, \\ R. Diehl ${ }^{\mathrm{f}}$, M. McConnell ${ }^{\mathrm{g}}$, R. S. Miller ${ }^{\mathrm{g}}$, G. Rank ${ }^{\mathrm{f}}$, J. M. Ryan ${ }^{\mathrm{g}}$, \\ V. Schoenfelder ${ }^{\mathrm{f}}, \mathrm{C}$. Winkler ${ }^{\mathrm{c}}$ \\ ${ }^{a}$ Emergent-IT, Inc., Goddard Space Flight Center \\ ${ }^{b}$ Bridgewater State College \\ ${ }^{c}$ European Space Research and Technology Center \\ ${ }^{d}$ Eureka Scientific \\ ${ }^{e}$ University of Bern \\ ${ }^{f}$ Max Planck Institute for Extraterrestrial Physics \\ ${ }^{g}$ University of New Hampshire
}

\begin{abstract}
The "Pre-SMM" (Vestrand and Miller 1998) picture of gamma-ray line (GRL) flares was that they are relatively rare events. This picture was quickly put in question with the launch of the Solar Maximum Mission (SMM). Over 100 GRL flares were seen with sizes ranging from very large GOES class events (X12) down to moderately small events (M2). It was argued by some (Bai 1986) that this was still consistent with the idea that GRL events are rare. Others, however, argued the opposite (Vestrand 1988; Cliver, Crosby and Dennis 1994), stating that the lower end of this distribution was just a function of SMM's sensitivity. They stated that the launch of the Compton Gamma-ray Observatory (CGRO) would in fact continue this distribution to show even smaller GRL flares. In response to a BACODINE cosmic gamma-ray burst alert, COMPtonTELescope on the CGRO recorded gamma rays above $1 \mathrm{MeV}$ from the $\mathrm{C} 4$ flare at 0221 UT 20 January 2000. This event, though at the limits of COMPTEL's sensitivity, clearly shows a nuclear line excess above the continuum. Using new spectroscopy techniques we were able to resolve individual lines. This has allowed us to make a basic comparison of this event with the GRL flare distribution from SMM and also compare this flare with a wellobserved large GRL flare seen by OSSE.
\end{abstract}

On January 20, 2000, a GOES C4.1 class solar flare occurred. The soft X-ray flux began at $8640 \mathrm{~s}(02: 21 \mathrm{UT})$ peaked at $8760 \mathrm{~s}(02: 26 \mathrm{UT})$ and ended at $9000 \mathrm{~s}(02: 30$ UT). There was no H $\alpha$ identification but the Nobeyama radio telescope observed a radio burst from the flare at $\mathrm{N} 15 \mathrm{~W} 33$ corresponding to NOAA active region number 8829. COMPtonTELescope's rapid gamma ray burst response system was triggered by BATSE at $8642 \mathrm{~s}$. The trigger from BATSE also alerted OSSE, which subsequently slew its four detectors to the direction of the Sun.

The automated system of COMPTEL imaged the Sun at a significance of $7.2 \sigma$ using 94 events recorded from annuli within $1^{\circ}$ of the Sun. Significant emission was detected from approximately $8640 \mathrm{~s}$ to $8740 \mathrm{~s}$ (Figure 1). There is evidence in the energy loss spectrum for nuclear line emission from $\sim 1-10 \mathrm{MeV}$ (Figure 2). The flare was only observed in the telescope mode, no emission was detected in the raw or processed burst data (see Schönfelder et al. 1993 for a discussion of COMPTEL).

CP587, GAMMA 2001: Gamma-Ray Astrophysics 2001, edited by S. Ritz et al. (C) 2001 American Institute of Physics 0-7354-0027-X/01/\$18.00 


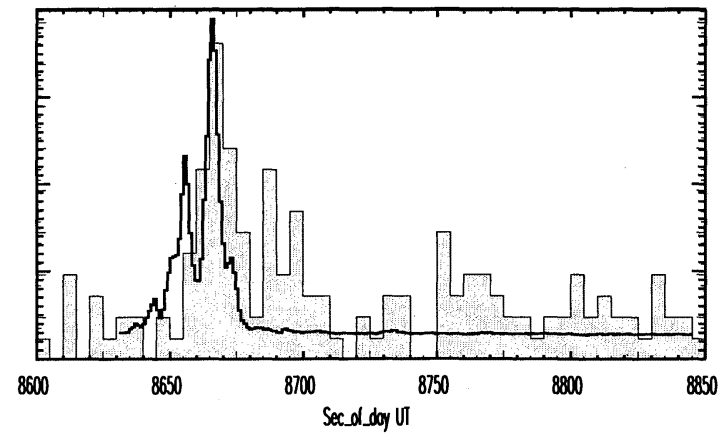

FIGURE 1. Light curves of the 20 January 2000 events observed in BATSE 1024 ms data (solid line) and the COMPTEL telescope (histogram).

OSSE observed emission above one MeV during the time from $8663 \mathrm{~s}$ to $8696 \mathrm{~s}$ (Murphy 2000). A single power law with photon spectral index $-2.85 \pm 0.3$ (Murphy 2000) provided an adequate fit to the OSSE data with no evidence of nuclear line emission. The total fluence above $50 \mathrm{keV}$ and $1 \mathrm{MeV}$ was $86 \pm 1$ and $0.8 \pm 0.6$ photons $\mathrm{cm}^{-2}$, respectively (Murphy 2000). The OSSE data place a 2- $\sigma$ upper limit for the $2.223 \mathrm{MeV}$ neutron capture line of 0.4 photon $\mathrm{cm}^{-2}$.

We selected the analysis interval for the COMPTEL telescope data to be 8640 $\mathrm{s}$ to $8740 \mathrm{~s}$. Normally, the background is modeled by using the average of 15 and 16 orbits before and after the event so that the geomagnetic conditions during the flare are similar. However, due to large data gaps only data 15 orbits before the flare are available. This limits the ability to produce the most accurate background model. Consequently, a different approach to background estimation was necessary. The next reasonable choice was to choose intervals just before and just after the flare as the background estimate.
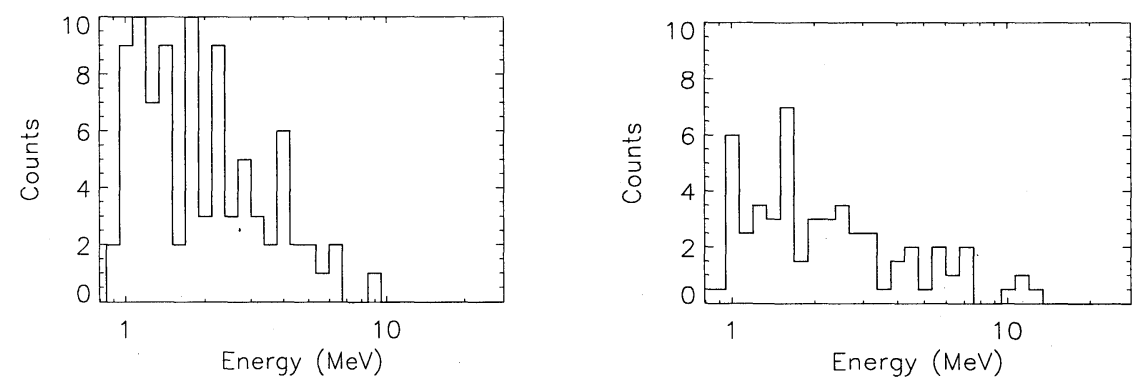

FIGURE 2. COMPTEL energy loss spectrum and background energy loss spectrum of the 20 January 2000 solar flare.

After a suitable background was selected, the energy loss spectrum (Figure 2a) and the selected background (Figure $2 \mathrm{~b}$ ) were deconvolved (using a new deconvolution technique, see Young et al. 2001) with the instrument response to obtain an estimate of the flux spectrum. In order to deal effectively with few counts in the energy loss 
spectrum at energies greater than $\sim 8 \mathrm{MeV}$, two energy binning were used. The flux spectrum was computed for a binning of 32 energy bins and 8 energy bins. These two were then combined, using the finer binning up to $8 \mathrm{MeV}$ and the coarser binning greater than $8 \mathrm{MeV}$.

Ideally, one would fit a gamma-ray spectrum with the individual components of the spectrum varying all parameters. Even for a large, intense event such as the June 4, 1991 event, COMPTEL does not have the statistics to resolve all the components such as the broad lines. A first approach even for a large event is to fit the expected strong lines and a composite spectrum for the remaining lines and continuum, varying only the amplitude of the lines and the composite spectrum. However, for the case of the January 20 flare, the fitting process is more difficult due the small number of counts.

Our approach to obtaining a reasonable model fit was to use data from the BATSE instrument to estimate the intensity and shape of the bremsstrahlung continuum. Using the most solar-facing BATSE detector, the data from $30 \mathrm{keV}$ to $1000 \mathrm{MeV}$ was fit with a broken power law with a first index of $-3.13 \pm 0.5$, a break energy of $86.7 \mathrm{keV}$ and a second index of $-2.85 \pm 0.02$ yielding a continuum flux above $1 \mathrm{MeV}$ of $(3.2 \pm 0.2) \times 10^{-3} \gamma \mathrm{cm}^{-2} \mathrm{~s}^{-1}$. The higher energy power law for the BATSE data is consistent with the fit obtained by OSSE.

In addition to the BATSE based power law continuum, different combinations of several nuclear components were tested. Eight different combinations were used. The two standard components of all the models were the power law from BATSE and a narrow line of unknown strength at $2.223 \mathrm{MeV}$. The best fit model (Figure 3) contained the addition of 3 narrow lines at $1.1,1.8$, and $4.4 \mathrm{MeV}$ to account for the 3 strongest features in addition to the $2.2 \mathrm{MeV}$ line (These four lines were determined significant with a P-value of $\left.3 \times 10^{-3}(3 \sigma)\right)$ by calculating the probability, under the assumption the mean rate of the source is zero, of obtaining as many events as observed or more given the background (Cowan 1998)). The model also included a composite spectrum of previously identified broad lines (Share and Murphy 1995).

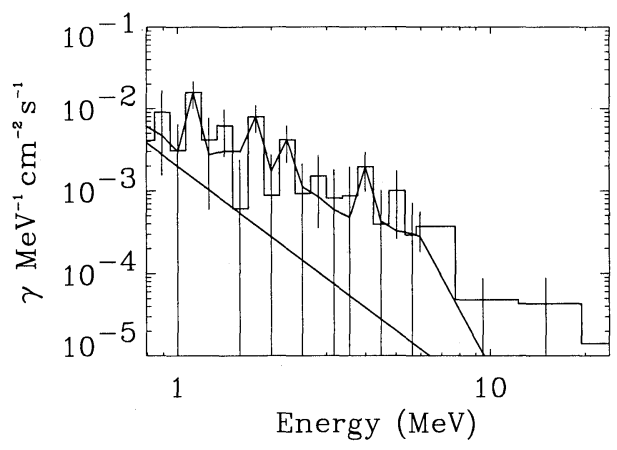

FIGURE 3. The best-fit model for the January 20, 2000 event. The model consists of a power law based on the BATSE data fit, a broad line template, and lines at 1.1,1.8,2.2, and 4.4 MeV. The straight solid line is the power law fit and the other solid line is the complete model. (Error bars extending to the bottom of the plots are $1 \sigma$ upper limits.) 
Using the results from the OSSE solar flare web page (Murphy 2000) and those of Murphy et al. 97, we can compare this small flare (model 2) to the X12+ class flare from 4 June 1991. These values are shown in Table 1.

TABLE 1. A comparison of the COMPTEL measurement of the January 202000 C4 GOES event and the OSSE measurement of the June 41991 X12+ GOES event.

\begin{tabular}{lll}
\hline & \multicolumn{1}{c}{ 2000 January 20 } & \multicolumn{1}{c}{ 1991 June 4 } \\
\hline $2.223 \mathrm{MeV}$ Fluence & $0.23 \pm 0.1$ & $1050 \pm 19$ \\
$4.4 \mathrm{MeV}$ fluence & $1.28 \pm 0.1$ & $189 \pm 9$ \\
$>1 \mathrm{MeV}$ fluence & $0.813 \pm 0.67$ & $\sim 3500 \pm 63$ \\
$2.223 / 4.4$ fluence ratio & $0.18 \pm 0.14$ & $5.56 \pm 0.28$ \\
Proton spectral index & $>5.5(\alpha / \mathrm{p}=0.1)$ & $3.37 \pm 0.1(\alpha / \mathrm{p}=0.1)$ \\
(Ramaty 1996) & & \\
$2.223 />1 \mathrm{MeV}$ fluence ratio & 0.24 & $\sim 0.3$ \\
$\#>30 \mathrm{MeV}$ protons & $1 \times 10^{31}$ & $(6.7 \pm 1.2) \times 10^{32}$ \\
\hline
\end{tabular}

One final important comparison for this flare is with the set of gamma-ray line events observed by SMM. SMM was pivotal in dispelling the idea that gamma-ray line emission was rare and only occurred in the largest of flares. However, due to its sensitivity SMM never had a positive detection of nuclear lines from any of the $\mathrm{C}$ class flare that it observed. COMPTEL, which is roughly an order of magnitude more sensitive than SMM has extended the SMM distribution of gamma ray flares by roughly an order of magnitude smaller. Figure 4 shows the SMM distribution of flare narrow nuclear line fluence as a function of the continuum power law fluence and GOES soft X-ray class (Vestrand et al. 1999).

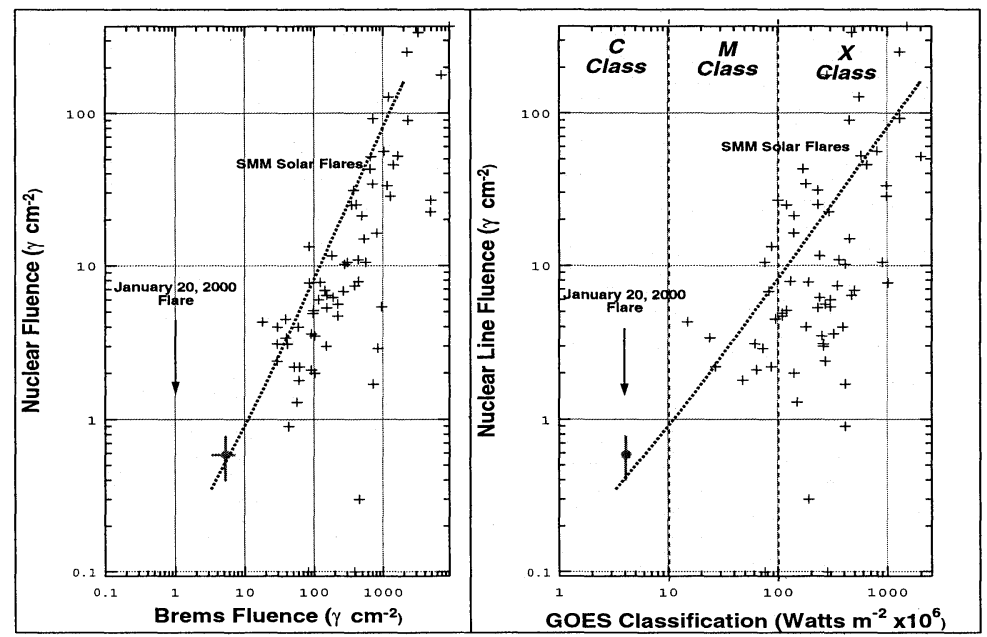

FIGURE 4. Scatter plots of narrow nuclear line fluence vs. continuum fluence and GOES classification respectively for the SMM GRS catalog and the 20 January 2000 event observed with COMPTEL.

All of these comparisons indicate that as compared to the June 4, 1991 flare and the SMM observed gamma-ray flares, the solar flare on January 20, 2000 is a normal or ordinary gamma-ray line solar flare. Actually, this comparison has shown that the 4 
June 1991 flares is the odd flare of the bunch. It shows an unusually large electron bremsstrahlung component.

In response to a BACODINE cosmic gamma-ray burst alert, COMPTEL on the CGRO recorded gamma rays above $1 \mathrm{MeV}$ from the C4 flare at 0221 UT 20 January 2000. This event, though at the limits of COMPTEL's sensitivity, clearly shows a nuclear line excess above the continuum. Using the new spectroscopy techniques we are able to resolve individual lines. This has allows us to make a basic comparison of this event with the GRL flare distribution from SMM and also compare this flare with a well-observed large GRL flare seen by OSSE. We show that this flare is normal, i.e., it is a natural extension of the SMM distribution of flares. The analysis of this flare means there is no evidence for a lower flare size for proton acceleration. Protons even in small flares contain a large part of the accelerated particle energy.

\section{ACKNOWLEDGMENTS}

This work was supported through NASA contract NAS5-26645 and NASA's Supporting Research and Technology program.

\section{REFERENCES}

1. Bai, T., Ap. J. 308, 912 (1986).

2. Cliver, E. W. et al., Ap. J. 426, 767-773 (1994).

3. Cowen, G., Statistical Data Analysis, Oxford: Claredon Press, 1998, p. 59.

4. Murphy R. J. et al., Ap. J. 490, 883-900 (1997).

5. Murphy, R. J., http://osse-www.nrl.navy.mil/solarflare/flarelib.htm,2000.

6. Ramaty, R. et al., "Solar Atmospheric Abundances from Gamma Ray Spectroscopy" in High Energy Solar Physics Workshop, edited by R. Ramaty et al., AIP Conference Proceedings 374, New York: American Institute of Physics, 1996, pp. 172-183.

7. Schönfelder, V. et al., Ap. J. Suppl. Series 86, 657-692 (1993).

8. Share, G.H. and Murphy, R. J., Ap. J. 452, 933 (1995).

9. Vestrand, W. T., Solar Phys 118, 95 (1988).

10. Vestrand, W. T., and Miller, J. A., "Particle Acceleration in Flares," in The Many Faces of the Sun: A Summary of the Results from NASA's Solar Maximum Mission, edited by K. T. Strong et al., New York City: Springer-Verlag, 1998, pp. 231-272.

11. Vestrand, W. T. et al., Ap. J. Suppl. Series 120, 409-467 (1999).

12. Young, C. A. et al., "Bayesian Multiscale Deconvolution Applied to Gamma-ray Spectroscopy" in These Proceedings, 2001. 